Bei einem relativ hohen $\mathrm{Cu}$ - und S-Gehalt eines Eisens wäre es ja möglich, dass beide Bestimmungen fehlerhaft ausfallen können, da sich vielleicht in Salzsäure unlōsliches Cu S bilden dürfte. - Brugmann (Chem. N. 54, 290) hat nachgewiesen, dass bei der Bestimmung des $\mathrm{S}$ im Eisen durch Lösen in $\mathrm{H} \mathrm{Cl}$ die Gegenwart von $\mathrm{Cu}$ unschädlich ist, wenn der Gebalt des letzteren unter 1 Proc. beträgt.

Zur Bestimmung des Mangans in Eisenlegirungen bedient man sich selten der gewichtsanalytischen Methode, am allerwenigsten wird man das Mn als Sulfid bestimmen, sondern zieht die Bestimmung als Pyrophosphat, wie dieselbe von $\mathrm{G} 00 \mathrm{ch}$ und A ustin (Z. f. anorg. Ch. 1898, 18, 339) beschrieben ist, vor. Man wägt allerdings wohl das Mangan als $\mathrm{MnS}$, erbält letzteres aber nicht etwa durch Fällen mit Schwefelammon, sondern fübrt das als Superoxydhydrat gefällte Mangan durch Reinigen und Glüben in $\mathrm{Mn}_{3} \mathrm{O}_{4}$ über, und dieses Oxyd verwandelt man dann durch Glühen mit Schwefel im Rose'schen Tiegel in Schwefelmangan. Diese Methode hat R ürup (Chemzg. 1896, 285) in seiner Arbeit: "Vergleichende Manganbestimmungen in Stahl und Eisen" ausfübrlich beschrieben.

Nun eignen sich aber alle gewichtsanalytischen Mn-Bestimmungen, der langen Zeitdauer wegen, nicht für die Praxis im Eisenhütten-Laboratorium; hier benutzt man die maassanalytische Methode nach VolhardWolff (Led ebur, Leitf. 1895, S. 79), welche bequem in einer Stunde ausführbar ist und genügend genaue Resultate gibt; ich mache aber darauf aufmerksam, dass der Factor für den Mangantiter nicht 0,2946 , wie Ledebur angibt, sondern 0,308 (Fischer's Jahresber. 1898, 120) ist. Von der Richtigkeit dieser letzteren Zahl.habe ich mich durch mehrere Controlanalysen überzeugt.

Neues Pyknometer, speciell für die Bestimmung des specifischen Gewichtes leichter Flüssigkeiten.

$$
\text { Von }
$$

Dr. Heinrich Göckel.

(Mittheilung aus dem chemischen Laboratorium der Thüringischen Glasinstrumentenfabrik von Alt, Eberhardt und Jäger in Ilmenau.)

Sämmtliche existirenden Pyknometerfläschchen haben bei ihrer Beschickung mit specifisch leichten Flüssigkeiten den grossen Nachtheil, dass dieselben zum Temperiren in ein Gefäss mit Wasser gebracht über- haupt nicht stehen oder äusserst leicht umkippen und müssen daher mit Klemmen befestigt oder auf irgend eine Weise, z. B. mit einem Bleiring beschwert werden. Es sind dies Manipulationen, die zumal bei schnellem Arbeiten mit einer grösseren Anzahl von Pyknometern immerhin umständlich und lästig sind. Oft werden überhaupt keine passenden Klemmen oder geeignete Beschwerungsmassen vorbanden sein.

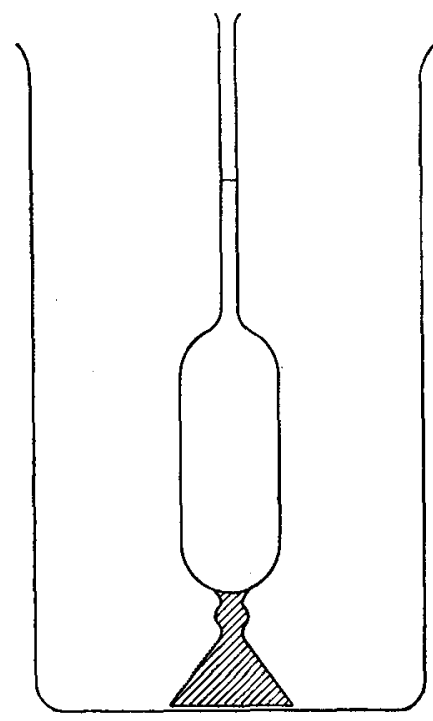

Fig. 302.

Das neue Pyknometer, dem eine grössere Stabilität gegeben ist, soll nun diesem Übelstande abhelfen. Wie aus Fig. 302 ersichtlich, ist dasselbe mit einem massiven polirten Glasfuss versehen worden, dessen Gewicht so ermittelt ist, dass das Pyknometer nicht unnöthig schwer wird und doch Masse genug besitzt, um mit Flüssigkeiten von sehr niedrigen specifischen Gewichten, wie Petroleumäther, Gasolin, Benzin, Ligroin, Äther u. s. w. gefüllt in einem Gefäss mit Wasser vollständig sicher zu stehen. Das Pyknometer ist selbst zu Bestimmungen des specifischen Gewichtes schwerer Flüssigkeiten zu empfehlen, indem die bisherigen mit solchen Flüssigkeiten gefüllten Pyknometer in nicht in Bewegung befindlichem Wasser zwar ruhig stehen, beim Umrühren desselben behufs Erreichung einer gleichmässigen Temperatur jedoch sehr leicht umkippen können. Die neue Form des Pyknometers bedingt zugleich einen weiteren Vortbeil, der darin besteht, dass der Pyknometerkörper allseitig mit Wasser in Berührung gebracht werden kann, dessen Temperatur derselbe annehmen soll. Es wird also bei demselben eine schnellere Temperirung erreicht als bei den bisherigen Pyknometern in Flaschenform, deren Bodenfiâche mit dem Wasser überbaupt nicht oder 
nur zum Theil in Berübrung kommen kann, wenn dieselben auf einen Siebboden gestellt werden ${ }^{1}$ ).

\section{Jodometrische Methode zur Untersuchung} von Chlorat-Hypochlorit-Gemischen.

$$
\text { Von }
$$

Hugo Ditz und Heinrich Knöpfelmacher.

Die Methoden und Vorschläge um Cblorate neben Hypochloriten zu bestimmmen, beruhen entweder auf der Reduction des Cblorates zu Cblorid und Ermittelung des letzteren auf gewichtsanalytischem oder volumetrischem Wege oder auf der maassanalytischen Bestimmung des Überschusses des in bestimmter Menge zugesetzten Reductionsmittels, oder endlich bestimmt man die Menge des aus dem Reductionsmittel entstandenen Oxydationsproductes.

Alle diese Methoden unterscheiden sich von einander einerseits durch die Art des Reductionsvorganges und -mittels, andererseits dadurch, dass das Chlorat entweder nach Zerstörung des Hypochlorits direct ermittelt wird, oder nach der Bestimmung der Summe des Hypochlorits und ChloratChlors bez. auch des Chlorid-Chlors das für sich bestimmte Hypochlorit- bez. auch Chlorid-Chlor in Abzug gebracht, also das Chlorat indirect gefunden wird.

$\mathrm{Zu}$ den Methoden, die auf der Bestimmung des nach der Reduction rorbandenen Chlorid-Chlors beruhen, gehört die ron Pattinson ${ }^{1}$ ) angegebene zur Aoalyse von Chlorkalk. Derselbe reducirt das Hypochlorit und Chlorat durch schweflige Säure und bestimmt nun nach Entfernung des Ūberschusses des Reductionsmittels das gesammte als Chlorid vorbandene Chlor und subtrahirt hiervon die Summe des bekannten Hypochlorit- und Cblorid-Chlors. Ganz auf demselben Principe berubt das Verfahren von L. M. Norton ${ }^{2}$ ) zur Analyse von elektrolysirten Chloridlósungen. Bei der Analyse zersetzter Chlorkalke zerstört $\mathrm{Opl}^{3}$ ) durch Kochen mit Ammoniak das Hypocblorit, bestimmt dann durch Titration mit Silberlösung alles Chlor mit Ausnahme desjenigen der Chlorsāure und ermittelt nach dem Glühen der Substanz das gesammte Chlor

1) Das hier beschriebene stabile Pyknometer ist in sauberster Ausführung und in bester Justirung mit jedem gewünschteu Inhalt von obengenannter Firma zu beziehen, welche den Apparat unter Gebrauchsmusterschutz stellen wird.

1) J. Soc. Chem. Ind. 1888, 188.

2) D. Zft. $1893,23$.

3) Dingler, (1875) 215, 239.

4) Zft. anorg. Ch. 13, 385 . des Chlorkalks. Bhaduris) reducirt durch ein Kupfer-Zink-Paar und bestimmt das gebildete Chlorid gewichtsanalytisch oder volumetrisch, wāhrend Rasennack ${ }^{5}$ ) diese Reduction mittels Zink vornimmt.

Die Bestimmung des Überschusses des Reductionsmittels erfolgt bei einem ebenfalls ron $B h$ adu ri (a. a. O.) angegebenen Vorschlage, nach welchem die Reduction mit Stannochlorid in salzsaurer Lösung erfolgt und der Überschuss desselben mit Permanganat bestimmt wird. Nach Lunge ${ }^{6}$ ) wird das Chlorat bestimmt, indem man durch Kochen mit Eisenvitriol unter Zusatz von Schwefelsāure und Zurücktitriren mit Chamāleon die Summe ron bleichendem Cblor und Chlorat-Chlor ermittelt und hiervon die Menge des bleichenden Chlors subtrabirt, welch' letzteres chlorometrisch oder, wie dies Oettel angibt, jodometrisch bestimmt wird. Nach Foerster und Jorre ${ }^{7}$ ) ist dieses Verfahren bei Gegenwart von wenig Hypochlorit ein recht genaues. Im Falle aber grössere Mengen des letzteren vorhanden waren, fanden sie ähnliche Schwierigkeiten, wie sie schon Woblwill ${ }^{8}$ ) erörtert hatte und welche darin bestehen, dass beim Mischen der hjpochloritbaltigen Lösung mit saurer Eisenlösung stets wahrnehmbare Mengen unterchloriger Sãure bez. Chlor entweichen ganz abgeseben von der Unannehmlichkeit des Arbeitens mit reiner Eisenvitriollösung. Bhaduri (a. a. O.) nimmt daber die Reduction mit Eisenoxydulsalz in alkalischer Lōsung vor, welche Art der Reduction aber nach Wohlwill (a. a. 0.) den Übelstand hat, dass das durch Oxydation entstandene Ferribydroxyd in scbwer löslicher Form auftritt, dasselbe durch lang andauerndes Kochen mit Salzsäure zwar in Lōsung gebracbt wird, die Lösung aber colloidal ist, beim Erkalten wieder einen Niederschlag absetzt und von so ungünstiger Farbe ist, dass das Titriren unmöglich ist. Auf dieses Verbalten des hier gebildeten Ferrihydroxydes weist auch schon Mendelejeff hin $\left.{ }^{9}\right)$. Wohlwill schlägt duher vor, vor der Reduction mit Eisenvitriollösung das Hypocblorit zu zerstören, indem man dasselbe in einen mit verdünnter Schwefelsäure gefüllten engen Glascylinder giesst und das entstebende Chlor durch einen kräftigen Luftstrom verjagt. $\mathrm{Zur}$ quantitativen $\mathrm{Be}$ stimmung eines Gemenges von Chlorid, $\mathrm{Hy}-$ pochlorit und Chlorat titrirt A. Carnot ${ }^{10}$ )

5) Dammer's Lex. d. Verfälschg. S. 423.

6 Handb. d. Sodaind., 2. Aufl. 3. Bd., S. 385.

T) J. f. prakt. Ch. $1899,58$.

${ }^{8}$ Z. f. Elektrochemie 5, 63 .

9) Grundlagen der Chemie, S. 1018.

10) Z. anorg. Chemie B. 15, 371 . 